Supplemental Information for

\title{
Carbon/Molecule/Metal and Carbon/Molecule/Metal Oxide Molecular Electronic Junctions
}

Rajendra Prasad Kalakodimi

Aletha M. Nowak

Richard L. McCreery*

The Ohio State University

Department of Chemistry 100 West $18^{\text {th }}$ Avenue Columbus $\mathrm{OH} 43210$

* corresponding author

614-292-2021

mccreery.2@osu.edu 


\section{Detailed discussion of XPS results:}

As reported previously ${ }^{1}$, the $\mathrm{TiOx}$ layer formed using a chamber pressure in the range of $2 \times 10^{-7}$ to $8 \times 10^{-6}$ torr is a mixture of $\mathrm{Ti}^{\mathrm{o}}$ and $\mathrm{Ti}^{\mathrm{II}}, \mathrm{Ti}^{\mathrm{III}}$ and $\mathrm{Ti}^{\mathrm{IV}}$ oxides. The oxide level strongly affected the electronic behavior, but its effects were avoided by substituting $\mathrm{Cu}$ for Ti. Given the importance of oxides to junction behavior, the various junction configurations used in the current work were examined with XPS depth profiling. In all cases, the metal or metal oxide layer was nominally $3.0 \mathrm{~nm}$ thick, and had a protective Au layer $7.0 \mathrm{~nm}$ thick. The XPS spectra shown in Figures 4 and 5 and the binding energies listed in Table $1 \mathrm{~S}$ were obtained when the survey spectra indicated that $\mathrm{Ar}^{+}$etching had progressed to the metal/molecule or metal oxide/molecule interface.

High resolution scans of the NAB/AlOx interfacial region are shown in Figure 4, as are those from $\mathrm{FL} / \mathrm{Cu}$ junctions. The single $\mathrm{Al}_{2 \mathrm{p}}$ peak observed at $75.4 \mathrm{eV}$ can be assigned to either $\mathrm{Al}_{2} \mathrm{O}_{3}$ or $\mathrm{Al}(\mathrm{OH})_{3}$. The literature values for the $\mathrm{Al}_{2 \mathrm{p}}$ binding energy of aluminum oxides and hydroxides are separated by $\sim 1 \mathrm{eV}$, thus a definite identification is difficult from the $\mathrm{Al}_{2 \mathrm{p}}$ band alone. The $\mathrm{O}_{1 \mathrm{~s}}$ XPS spectrum collected at the same depth as the $\mathrm{Al}_{2 \mathrm{p}}$ spectrum is also included in Figure 4. The primary oxygen component is consistent with a metal hydroxide species $(531.6 \mathrm{eV})^{2-7}$. A minor component detected at $534.0 \mathrm{eV}$ can be attributed to water ${ }^{8}$, and possibly a small contribution of metal oxide at $530.7 \mathrm{eV}^{4,9,10}$. There is no evidence for the formation of an $\mathrm{Al}-\mathrm{N}$ bond between the vapor deposited $\mathrm{Al}$ and the $\mathrm{NO}_{2}$ groups in the $\mathrm{NAB}$ layer, due to the absence of an $\mathrm{Al}_{2 \mathrm{p}}$ peak at $\sim 74.5 \mathrm{eV}^{8,11}$ and an $\mathrm{N}_{1 \mathrm{~s}}$ peak at $\sim 397 \mathrm{eV}^{12-14}$. After further etching, a single $\mathrm{N}_{1 \mathrm{~s}}$ peak was detected at $399.3 \mathrm{eV}$, which is characteristic of the $\mathrm{N}=\mathrm{N}$ group in the NAB (Fig.4) ${ }^{15}$. 
Overall, the XPS results clearly establish that the $\mathrm{Al}$ layer is $\mathrm{Al}^{\mathrm{III}}$, mostly as hydroxide, which does not appear to interact strongly with the NAB layer.

The lower row of spectra in Figure 4 were obtained near the fluorene/Cu interface in a PPF/FL(1.7)/Cu(3.0)/Au junction following $\mathrm{Ar}^{+}$sputtering. At no point after the initiation of depth profiling was oxygen detected for the PPF/FL/Cu/Au junction. The high-resolution spectrum of the $\mathrm{Ti}_{2 \mathrm{p}}$ and $\mathrm{O}_{1 \mathrm{~s}}$ regions of $\mathrm{NAB}$ (4.5)/TiOx junctions made with different Ti deposition conditions are shown in Figure 5. The upper spectra in Figure 5 were obtained with the "TiOx" conditions in which the Ti was not exposed to air before Au deposition. While minimal $\mathrm{Ti}^{\circ}$ was observed at $453.9 \mathrm{eV}$, the predominant titanium species was $\mathrm{Ti}_{2} \mathrm{O}_{3}$, which is responsible for approximately $42 \%$ of the total $\mathrm{Ti}_{2 \mathrm{p}}$ signal ${ }^{10,14}$, while $\mathrm{TiO}_{2}$ accounted for $\sim 34 \%$. The remaining Ti species, which is detected at $455.2 \mathrm{eV}$, comprises $\sim 24 \%$ of the $\mathrm{Ti}_{2 \mathrm{p}}$ envelope and can be assigned to either Ti-N or $\mathrm{TiO}\left(2 \mathrm{p}_{3 / 2}\right)^{8,10,16-18}$. Deconvolution of the $\mathrm{O}_{1 \mathrm{~s}}$ revealed three oxygen species present within the titanium layer, that of metal oxide $(530.7)^{4,9,10}$, metal hydroxide $(531.6)^{2,4-7}$, and an unidentified peak at $532.2 \mathrm{eV}$. Two peaks were observed in the $\mathrm{N}_{1 \mathrm{~s}}$ region, which correspond to the $\mathrm{N}=\mathrm{N}$ group in the NAB layer $(399.3 \mathrm{eV}){ }^{15}$ and the Ti-N bond between the $\mathrm{NO}_{2}$ group and the titanium top contact (397.1) ${ }^{17-20}$. The formation of this covalent Ti-N bond was established in a previous study ${ }^{19}$, and implies a molecular junction with covalent bonds at both the Ti-NAB and PPF-NAB interfaces.

As noted previously ${ }^{1}$, reduction of backpressure during Ti deposition reduces, but does not eliminate Ti oxides, at least for the minimum backpressure available with the apparatus used ( $2 \times 10^{-7}$ torr). However, increasing the pressure and exposing the Ti deposit to air results in a $\mathrm{Ti}$ layer which is predominately $\mathrm{TiO}_{2}$. The lower spectra of 
Figure 5 were obtained from a $\mathrm{PPF} / \mathrm{NAB}(4.5) / \mathrm{TiO}_{2}(3.1) / \mathrm{Au}$ junction after $\mathrm{Ar}^{+}$etching to reach the $\mathrm{NAB} / \mathrm{Ti}$ interface. All observable $\mathrm{Ti}$ is present as $\mathrm{Ti}^{\mathrm{IV}}$ and the $\mathrm{O}_{1 \mathrm{~s}}$ spectrum corresponds to metal oxide rather than hydroxide. Whenever Ti junctions are discussed, they have been prepared such that $\mathrm{Ti}$ occurs as $\mathrm{TiO}_{2}$, unless noted otherwise. XPS of $\mathrm{PPF} / \mathrm{NAB}(4.5) / \mathrm{Cu} / \mathrm{Au}$ junctions (data not shown) indicated low levels of oxygen at the copper/molecule interface, some of which originated in the NAB nitro group.

\section{Table 1S:}

Observed Binding Energies and Assignments for PPF/molecule/metal/Au Junctions

\begin{tabular}{|c|c|c|c|c|}
\hline & $\begin{array}{c}\text { Binding Energy } \\
(\mathrm{eV}) \\
\text { observed }\end{array}$ & Assignment & $\begin{array}{c}\text { Binding Energy } \\
(\mathrm{eV}) \\
\text { Literature }\end{array}$ & References \\
\hline $\mathbf{N}_{1 \mathrm{~s}}$ & $\begin{array}{l}399.3 \\
397.1\end{array}$ & $\begin{array}{l}\mathrm{NO}_{2} \\
\mathrm{~N}=\mathrm{N} \\
\mathrm{Ti}-\mathrm{N} \\
\mathrm{Al}-\mathrm{N} \\
\mathrm{R}^{-N_{2}}{ }^{+} \\
\mathrm{R}^{-N_{2}}{ }^{+}\end{array}$ & $\begin{array}{c}406 \\
400 \\
396.9-397.4 \\
396.5-398.6 \\
405.1 \\
403.8\end{array}$ & $\begin{array}{c}15 \\
15 \\
17,18,20 \\
12,14,21 \\
22,23 \\
22\end{array}$ \\
\hline $\mathbf{T i}_{2 p}$ & $\begin{array}{l}458.5 \\
463.7 \\
456.5 \\
461.3 \\
455.2 \\
\\
455.2\end{array}$ & $\begin{array}{c}\mathrm{TiO}_{2}(2 \mathrm{p} 3 / 2) \\
\mathrm{TiO}_{2}(2 \mathrm{p} 1 / 2) \\
\mathrm{Ti}_{2} \mathrm{O}_{3}(2 \mathrm{p} 3 / 2) \\
\mathrm{Ti}_{2} \mathrm{O}_{3}(2 \mathrm{p} 1 / 2) \\
\mathrm{TiO}(2 \mathrm{p} 3 / 2) \\
\mathrm{TiO}(2 \mathrm{p} 1 / 2) \\
\mathrm{Ti}-\mathrm{N} \\
\mathrm{Ti}-\mathrm{C} \\
\mathrm{Ti}^{\circ}(2 \mathrm{p} 3 / 2)\end{array}$ & $\begin{array}{c}459.0-458.7 \\
464.4-464.5 \\
456.6-457.1 \\
461.8-462.8 \\
454.4-455.3 \\
460.0-460.9 \\
455.2-455.8 \\
454.6-454.7 \\
453.9\end{array}$ & $\begin{array}{c}4,10,16,24,25 \\
4,10,16 \\
10,16 \\
10,16 \\
10,16 \\
10,16 \\
817,18,20 \\
26,27 \\
8,16\end{array}$ \\
\hline $\mathbf{A l}_{2 p}$ & 75.4 & $\begin{array}{c}\gamma-\mathrm{Al}_{2} \mathrm{O}_{3} \\
\alpha-\mathrm{Al}_{2} \mathrm{O}_{3} \\
\mathrm{Al}_{2} \mathrm{O}_{3} \\
\mathrm{AlOOH} \\
\mathrm{Al}(\mathrm{OH})_{3} \\
\mathrm{Al}^{\circ} \\
\mathrm{Al}-\mathrm{N} \\
\mathrm{Al}-\mathrm{C}\end{array}$ & $\begin{array}{c}73.72 \\
73.85 \\
74.9-75.6 \\
74.22 \\
74.3 \\
72.85 \\
75.0-74.0 \\
73.4\end{array}$ & $\begin{array}{c}8 \\
8 \\
28-30 \\
8 \\
8 \\
8,28 \\
8,11 \\
11\end{array}$ \\
\hline
\end{tabular}




\begin{tabular}{|c|c|c|c|c|}
\hline $\mathbf{C}_{1 \mathrm{~s}}$ & 284.5 & $\begin{array}{l}\text { Ti-C } \\
\text { graphite } \\
\text { Al-C }\end{array}$ & $\begin{array}{c}281.5 \\
284.5 \\
282.5-283.4\end{array}$ & $\begin{array}{c}8,26,27 \\
8,31 \\
11,32\end{array}$ \\
\hline $\mathbf{O}_{1 \mathrm{~s}}$ & $\begin{array}{c}534.0 \text { (Al only) } \\
530.7 \text { (Ti only) } \\
531.6\end{array}$ & $\begin{array}{c}\text { water } \\
\text { metal oxide } \\
\text { metal } \\
\text { hydroxide }\end{array}$ & $\begin{array}{l}533.1 \\
530.5 \\
531.7\end{array}$ & $\begin{array}{c}8 \\
4,109 \\
2-4\end{array}$ \\
\hline $\mathbf{A} \mathbf{u}_{4 \mathrm{f}}$ & $\begin{array}{l}84.1 \\
87.8\end{array}$ & $\begin{array}{l}\mathrm{Au}\left(4 \mathrm{f}_{7 / 2}\right) \\
\mathrm{Au}\left(4 \mathrm{f}_{5 / 2}\right)\end{array}$ & $\begin{array}{l}84 \\
88\end{array}$ & \\
\hline $\mathrm{Cu}_{2 p}$ & $\begin{array}{c}932.5 \\
952\end{array}$ & $\begin{array}{l}\mathrm{Cu}\left(2 \mathrm{p}_{3 / 2}\right) \\
\mathrm{Cu}\left(2 \mathrm{p}_{1 / 2}\right)\end{array}$ & $\begin{array}{l}932.6 \\
952.5\end{array}$ & $\begin{array}{l}33 \\
33\end{array}$ \\
\hline
\end{tabular}

Table 2S. Metal Deposition Conditions

PPF/Molecule/Cu (3.0)/Au (7.0)

$\mathrm{Cu}(3.0 \mathrm{~nm})$

$\mathrm{Au}(7.0 \mathrm{~nm})$
Pressure, torr Rate, $\mathrm{nm} / \mathrm{sec}$

$\begin{array}{ll}3 \times 10^{-7} & 0.1 \\ 3 \times 10^{-7} & 0.1\end{array}$

0.1

PPF/Molecule/AIOx/Au

$\mathrm{Al}(3.3 \mathrm{~nm}) \quad 5 \times 10^{-6} \quad 0.03$

$\mathrm{Au}(7.0 \mathrm{~nm}) \quad 5 \times 10^{-6} \quad 0.1$

PPF/Molecule/TiOx/Au

$\mathrm{TiOx}(3.1 \mathrm{~nm})^{\mathrm{a}} \quad(0.5-4) \times 10^{-6} \quad 0.03$

$\mathrm{Au}(7.0 \mathrm{~nm}) \quad 4 \times 10^{-6} \quad 0.1$

PPF/Molecule/TiO $/$ /Au

$\begin{array}{rll}\text { TiOx }(3.1 \mathrm{~nm}) & 7 \times 10^{-6} & 0.03 \\ \text { vent with air } & \sim 760 & (45 \text { minutes }) \\ \mathrm{Au}(7.0 \mathrm{~nm}) & 4 \times 10^{-6} & 0.1\end{array}$

a. a range of deposition pressures was used, as discussed in the "experimental" in main text. 
References for "supplemental information":

(1) McGovern, W. R.; Anariba, F.; McCreery, R.; J. Electrochem Soc 2005, 152, E176.

(2) Wagner, C. D.; Zatko, D. A.; Raymond, R. H.; Anal. Chem. 1980, 52, 1445.

(3) McIntyre, N. S.; Sunder, S.; Shoesmith, D. W.; Stanchell, F. W.; J. Vac. Sci. Technol. A 1981, 18, 714.

(4) Moulder, J. F.; Stickle, W. F.; Sobol, W. F.; Bomben, K. D. Handbook of X-Ray Photoelectron Spectroscopy; Perkin-Elmer Corp., Physical Electronics Div.: Eden Prairie, 1992.

(5) Dickinson, T.; Povey, A. F.; Sherwood, P. M. A.; J. Chem. Soc. Faraday Transactions 1 1976, 72, 686.

(6) Haber, J.; Stoch, J.; Ungier, L.; J. Electron Spectrosc. Relat. Phenom. 1976, 9 , 459.

(7) Wagner, C. D.; Passoja, D. E.; Hillery, H. F.; Kinisky, T. G.; Six, H. A.; Jansen, W. T.; Taylor, J. A.; J. Vac. Sci. Technol. A 1982, 21, 933.

(8) Practical Surface Analysis, 2nd Edition, Vol. 1: Auger and X-ray Photoelectron Spectroscopy; Briggs, D.; Seah, M. P., Eds.; John Wiley and Sons: Chichester, 1990.

(9) Netterfield, R. P.; Martin, P. J.; Pacey, C. G.; Sainty, W. G.; McKenzie, D. R.; J. Appl. Phys. 1989, 66, 1805.

(10) Gonbeau, D.; Guimon, C.; Pfister-Guillouzo, G.; Levasseur, A.; Meunier, G.; Dormoy, R.; Surf. Sci. 1991, 254, 81.

(11) Ning, J.; Xu, S.; Ostrikov, K. N.; Chai, J.; Li, Y.; Koh, M. L.; Lee, S.; Thin Solid Films 2001, 385, 55.

(12) Liao, H. M.; Sodhi, R. N. S.; Coyle, T. W.; J. Vac. Sci. Technol. A 1993, 11, 2681.

(13) Zhang, J.; Anson, F. C.; J. Electroanal. Chem. 1992, 331, 945.

(14) Katnani, A. D.; Papathomas, K. I.; J. Vac. Sci. Technol. A 1987, 5, 1335.

(15) Liu, Y.-C.; McCreery, R. L.; Anal. Chem. 1997, 69, 2091.

(16) Saied, S. O.; Sullivan, J. L.; Choudhury, T.; Pearce, C. G.; Vacuum 1988, 38, 917.

(17) Badrinarayanan, S.; Sinha, S.; Mandale, A. B.; J. Electron Spectrosc. Relat. Phenom. 1989, 49, 303.

(18) Biwer, B. M.; Bernasek, S. L.; Surf. Sci. 1986, 167, 207.

(19) Nowak, A. M.; McCreery, R. L.; Anal. Chem. 2004, 76, 1089.

(20) Shulg'a, Y. M.; Troitskii, V. N.; Aivazov, M. I.; Borodk'o, Y. G.; Zh. Neorg. Khimii 1976, 21, 2621.

(21) Zhang, Y.; J. Mat. Sci. Lett. 2002, 21, 1603.

(22) Finn, P.; Jolly, W. L.; Inorganic Chemistry 1972, 11, 1434.

(23) Brant, P.; Feltham, R. D.; Journal of Organometallic Chemistry 1976, 120, C53.

(24) Slinkard, W. E.; DeGroot, P. B.; J. Catal. 1981, 68, 423.

(25) Murata, M.; Wakino, K.; Ikeda, S.; J. Electron Spectrosc. Relat. Phenom. 1975, 6, 459.

(26) Galuska, A. A.; Uht, J. C.; Marquez, N.; J. Vac. Sci. Technol. A 1988, 6, 110.

(27) MacInnes, A. N.; Barron, A. R.; Li, J. J.; Gilbert, T. R.; Polyhedron 1994, 13, 1315. 
(28) Kim, J. S.; Chakrabarti, K.; Lee, J.; Oh, K.-Y.; Lee, C. E.; Materials Chemistry and Physics 2003, 78, 733.

(29) Chowdhuri, A. R.; Takoudis, C. G.; Thin Solid Films 2004, 446, 155.

(30) Kim, D.-P.; Yeo, J.-W.; Kim, C.-I.; Thin Solid Films 2004, 459, 122.

(31) Ranganathan, S.; McCreery, R. L.; Anal. Chem. 2001, 73, 893.

(32) Ling, Q. D.; Li, S.; Kang, E. T.; Neoh, K. G.; Liu, B.; Huang, W.; Appl. Surf. Sci. 2002, 199, 74.

(33) Sun, Y. M.; Lee, S. Y.; Lemonds, A.; Lozano, J.; Zhou, J. P.; Ekerdt, J. G.; White, J. M.; Imesh, I.; Surf. Interface Anal. 2001, 32, 79. 